\title{
HOMO IMAGO DEI. LA INMANENCIA DEL DOGMA DE LA CREACIÓN EN LA CONSTRUCCIÓN CIENTÍFICA DE LA RELACIÓN ENTRE VARIACIÓN, DIFERENCIA Y DESIGUALDAD EN LA ESPECIE HUMANA. SIGLOS XVIII Y XIX
}

\author{
Mario César Sánchez Villa \\ Investigador Independiente \\ Email: mcsv1981@gmail.com \\ ORCID iD: http://orcid.org/0000-0002-4157-1059
}

Recibido: 16 mayo 2018; Aceptado: 2 noviembre 2019

Cómo citar este artículo/Citation: Sánchez Villa, Mario César (2020), “Homo imago Dei. La inmanencia del dogma de la creación en la construcción científica de la relación entre variación, diferencia y desigualdad en la especie humana. Siglos XVIII y XIX", Asclepio, 72(1): p301. https://doi.org/10.3989/asclepio.2020.10.

RESUMEN: Valiéndose de un análisis cultural de larga duración, el autor trata de analizar la influencia de la idea de la especificidad del ser humano dentro de la construcción de la historia natural en occidente. Se pretende mostrar cómo ese concepto del ser humano incidió en la construcción de los distintos razonamientos científicos sobre la relación entre variedad, diferencia y desigualdad, condicionando una forma de identidad biológica marcada por la apropiación del valor de superioridad física y moral esencial que se atribuyó al primer hombre. Ello da pie a una reflexión sobre la forma en que la relación entre la ciencia y el poder impuso límites al proceso de secularización en occidente.

PALABRAS CLAVE: Historia natural del ser humano; biología; secularización; religión; pecado original.

HOMO IMAGO DEI. THE IMMANENCE OF THE DOGMA OF CREATION IN THE SCIENTIFIC CONSTRUCTION OF THE RELATIONSHIP BETWEEN VARIATION, DIFFERENCE AND INEQUALITY IN THE HUMAN SPECIES. 18TH AND 19TH CENTURIES

\begin{abstract}
Through a cultural analysis in long-term, the author tries to analyze the influence of the idea of the specificity of the human being in the construction of Western natural history. The aim is to show how this concept of human being influenced the construction of the different scientific reasoning about the relationship between variety, difference and inequality, and how that conditioned the construction of a biological identity marked by the appropriation of the value of an essential physical and moral superiority attributed to the first man. This leads to a reflection on the way in which the relationship between science and power, imposed limits on the process of Western secularization.
\end{abstract}

KEY WORDS: Natural History of human being; Biology; secularization; religion; original sin. 
La relación por la que, dispuestos dos o más seres similares, se establece su inequidad y, conforme a cualquier tipo de criterio pretendidamente objetivo, se cataloga su diferencia, ha sido, es y siempre será, una relación subjetiva, dependiente de criterios tan universales como los que abarca la propia individualidad de quien, al juzgarlos, los hace valer como formas de poder biológico (Foucault, 2000). Históricamente, esta forma de poder tiende a justificarse sobre la consideración de que la variación entre términos de la misma naturaleza responde a diferencias consustanciales a esa misma naturaleza, hasta el punto de establecerse categorías distintas dentro de ella. Tal como lo advirtiera Gayo Plinio Segundo:

“... es un hecho breve de decir, pero infinito en su valoración, que hay tantos idiomas, tantas lenguas, tan gran diversidad de formas de hablar que un extranjero para el otro país apenas hace el papel de hombre" (Plinius Secundus, 77-79 ap., Lib. VII-1:7).

Sin embargo, la propia disposición del argumento indica que, si la evaluación de la variación es la constatación empírica de un hecho natural, su valoración como diferencia responde a una construcción cultural concreta que se extiende a lo largo del tiempo y que se "disfraza de verdad" conforme a intereses variables. No es de extrañar que a mediados del siglo XVIII Jean-Jaques Rousseau aún siguiera considerando confusos los criterios que reinaban en la explicación de las variaciones, dado que...

... después de trescientos o cuatrocientos años que los habitantes de la Europa inundaran las otras partes del mundo (...) estoy persuadido que (sic) nosotros no conocemos otros hombres más que los solos europeos, y aun parece (...) que cada uno no sabe casi otra cosa, bajo el nombre pomposo de estudio del hombre, que el de los hombres de su país (Rousseau, 1754, pp. 177-178).

Esta longue durée a la que se acoge el sentido de la diferencia en occidente, pone en evidencia el desprecio con el que históricamente ciertas formas de pensamiento racional se han apropiado de un concepto puro de la humanidad utilizándolo en su beneficio, para concederlo a discreción, sobre aquellas culturas o individuos que, a su juicio, fueran dignos de poseerla. La historiografía reciente, abierta a nuevas formas de pensamiento crítico con el poder es más consciente que nunca del papel principal, que desempeñó la ciencia en este proceso de construcción de la diferencia, necesario para legitimar los intereses políticos, económicos y sociales de los nuevos estados liberales modernos ${ }^{1}$, sin embargo, ha perdido de vista el papel que la propia tradición cul- tural de occidente tuvo en el sustento de esas formas "científicas" de prejuicio moderno (Cesaire, 1955).

A la hora de tratar sobre las diferencias entre los seres humanos, la ciencia de los siglos XVIII y XIX se limitó en gran medida a redescubrir, y en este sentido a demostrar, que había una inequidad natural entre los hombres, que estaba basada en razones morfológicas, y que sobre ella podía construirse una jerarquización y un derecho de opresión de unos por otros (Peset Reig, 1983, p. 9). No obstante, dentro del pensamiento científico racional la certeza o la plausibilidad de la mayor parte de estas cuestiones ya había sido asumida de modo dogmático desde mucho tiempo atrás.

La idea de este artículo es poner en evidencia los mecanismos culturales que explican la continuidad entre esas formas de pensamiento racional que unen al pensamiento dogmático o pre-científico con la ciencia. Dicho de otro modo, vamos a observar esas "formas de saber" que, sin llegar a ser ciencia, "dirigen la praxis de la vida humana (...) incluso cuando esta praxis se orienta ex professo a la promoción y aplicación de la ciencia" (Gadamer, 1960, p. 243). Para conseguirlo, nos vamos a centrar en un objeto concreto el principio del homo imago dei o la convicción profunda y constante en occidente de que, habiendo sido creado el ser humano a imagen y semejanza de Dios, y siendo la variedad una característica propia del ser humano, era el pueblo de occidente y no otro, el albacea de la forma original o esencia del individuo.

En este sentido, nuestra propuesta analítica pretende incidir sobre los límites del proceso de secularización en lo que se refiere a la condición humana. Creemos que frente a la idea de los cambios de paradigma de Kuhn (1962) o de los "magisterios separados" de Gould, no existió, respecto al ser humano, una forma de separación aséptica, ni tampoco una complementariedad de conocimientos al mismo nivel, entre la religión y la ciencia (Gould, 1999), sino una clara continuidad basada en intereses políticos e ideológicos comunes, que permitió que ciertas ideas "esotéricas" o "sobrenaturales" consiguieran sobreponerse o adaptarse al rigor creciente de un método científico que a lo largo de los siglos, fue perfeccionando su desarrollo crítico y racional, modificando la función que ejercía dicho conocimiento, pero jamás hasta el punto de cuestionarlo de modo efectivo, ni mucho menos definitivo (Durand, 1978).

El análisis de la diferencia desde un punto de vista antropológico no es algo nuevo ${ }^{2}$ y su uso conlleva ciertas carencias de las que es preciso advertir en 
este momento. La principal de todas ellas es que deforma, en cierta medida, la ambigüedad de la experiencia antropológica de la modernidad dentro del pensamiento occidental. Nuestro análisis es significativo a este respecto, pues partiendo del estudio del principio dogmático del homo imago dei va a centrarse únicamente en la construcción de formas de poder basadas en la idea de la desigualdad antropológica. En este sentido es sumamente importante no perder de vista que la influencia en la construcción del pensamiento científico moderno sobre la diferencia, estuvo íntimamente ligada al desarrollo de un pensamiento igualmente fructífero sobre el aspecto contrario, esto es, sobre la igualdad de todos los seres humanos y la unidad global del mundo ${ }^{3}$. Más allá de las evidentes razones de espacio, la decisión de centrarnos sólo en una parte de este pensamiento atiende a un interés concreto por observar, no tanto los argumentos antropológicos de la diferencia, como algunos de los recursos dialécticos o intelectuales que permitieron su utilización para la construcción de discursos de poder en el campo de la ciencia.

Ello explica a su vez la elección de una forma de análisis no menos problemática como es la longue durée. En general, un análisis de larga duración nos permitirá evaluar la influencia, persistencia y capacidad de adaptación del argumento religioso dentro de la construcción del pensamiento dogmático que nutrió los distintos razonamientos antropológicos de la diferencia en occidente. Sin embargo, ese mismo análisis no nos ofrecerá iguales garantías en lo referente al propio concepto de pueblo o cultura occidental, pues su definición depende en gran medida de las peculiaridades sociales, políticas o económicas de cada época, aspectos todos ellos en los que no podremos entrar en detalle si no es por el uso de métodos más concretos. Algo similar ocurre a la hora analizar la influencia de la religión, pues si, como acabamos de señalar, la elección de un análisis de larga duración resulta adecuada para comprobar la persistencia e influencia del argumento religioso sobre el científico, la propia idea de la fe resulta un concepto demasiado resbaladizo y dúctil, insuficiente en todo caso para explicar la construcción de razonamientos antropológicos sobre la diferencia y menos aún de su transformación o uso para el desarrollo de políticas concretas, como pueden ser las dirigidas a la colonización ${ }^{4}$.

Un análisis de estas cuestiones, requeriría de un enfoque y metodología distintos y de una forma de relato más densa, que permita atender con detalle al juego más amplio de fuerzas que definen la relación entre ciencia y religión en cada uno de los periodos que aquí simplemente sobrevolamos. La lounge dure otorga de este modo un relato histórico con cierta apariencia de linealidad y unidireccionalidad, una suerte de plano fijo a partir del cual se pretende mover a una reflexión histórica más profunda.

\section{PECADO Y VARIACIÓN. LA RACIONALIZACIÓN DEL MITO DE LA CREACIÓN Y LA CONSTRUCCIÓN DE LA DIFERENCIA ENTRE LOS SERES HUMANOS}

Parece lógico pensar que a la hora de construir relatos sobre el origen del mundo, las distintas culturas se hayan reservado como norma general un papel protagonista. A fin de cuentas, la idea de esos relatos no es sólo la de entretener, sino también la de transmitir una serie de valores que se ajustan a las necesidades de los que los cuentan y los escuchan, por lo que su disposición varía necesariamente de unas culturas a otras. En occidente, las distintas versiones del mito de las Edades del Hombre ${ }^{5}$ o los mitos de la tradición órfica del siglo VI AEC, plantearon diferentes visiones teogónicas de la creación dentro de una visión historicista de la naturaleza, en la que el ser humano había sido creado como una criatura superior al resto de seres mortales (imagen1). No es pre-

Imagen 1. Grabado de Heindrik Goltzius para ilustrar la edición de la Metamorfosis de Ovidio de Claes Janszoon Visscher (1589). La imagen narra en segundo plano el robo del fuego por parte del titán Prometeo. En primer plano, rodeado de varias especies animales, Prometeo usa el fuego robado para animar a Epimeteo, el primer hombre. Imagen cedida por http://www.britishmuseum.org

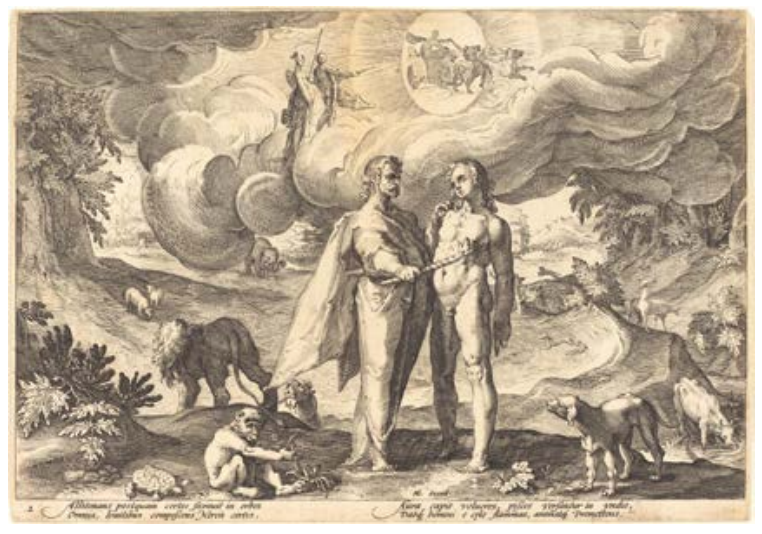


ciso introducirse en los debates históricos de la Religionswissenchaft decimonónica, para comprobar que el paralelismo entre estas mitologías y los relatos de la soteriología hebrea que sustentan al cristianismo, responde a una tradición común compartida con antiguas tradiciones orientales (Alter, 1996), pero es curioso observar cómo en su representación lógica o racional, los primeros derivaron hacia un modelo de historia natural marcado por la figura de un $\Delta \eta \mu \iota$ ovpós o maestro hacedor, que se limita a ordenar la materia preexistente, mientras que es un "Dios todopoderoso", capaz de crear ex nihilo, el que constituye el motor de la historia en los segundos (Herrero de Jáuregui, 2007) ${ }^{6}$.

Esta diferencia marcó distintas formas de entender el mundo que, no obstante, compartieron un similar concepto de la esencia humana. En ambos lados, el ser humano fue visto como una correlación de dos partes, una física o material, común al resto de animales, y otra anímica o inmaterial, mucho más acentuada en su especie. El orden de esa correlación se configuró como contingente del carácter imperfectible que se atribuyó a la entidad creadora (Estrada Díaz, 2000), siendo igualmente destacable que esa cualidad de la obra no se consideró extensible, al menos no necesariamente, a su descendencia, sino que su reproducción se vio condicionada a la concurrencia de diversos tipos de causas, dispuestos sobre dos grandes explicaciones: una ambientalista, que priorizó el efecto de los factores externos sobre las partes material y anímica (Kennedy y Jones-Lewis, 2016), y otra, espiritualista o moral en la que lo definitivo fue la capacidad del individuo para guiarse, o dejarse guiar, por comportamientos inadecuados a la calidad de su parte anímica o inmaterial, la cual no podía ser modificada de modo permanente, pero sí llegar a forzar cambios sobre la parte material o corporal que completaba al individuo (Gagné, 2013, pp. 80-158).

Una y otra explicación aceptaron que el grado de perfección esencial de origen llevaba aparejado un grado igualmente esencial de imperfección, cuya prevalencia explicaría los cambios y variaciones de la reproducción natural en los individuos. Puesta en concordancia con la especial correlación física y anímica del ser humano, la especulación sobre los mecanismos por los que se producía su reproducción, ofreció diversas explicaciones sobre el parecido o la diferencia de la descendencia con respecto a la forma de origen ${ }^{7}$. No obstante, en un orden estrictamente fisiológico esas explicaciones tardarían aun muchos siglos en salir del campo de lo especulativo y en ningún caso llegarían a establecer la actual relación entre los aspectos filogenéticos y ontogenéticos de la generación ${ }^{8}$.

En cualquier caso, es evidente que la especificidad del ser humano impide en cierto modo, aplicar el esquema tradicional con el que la historia de la biología ha analizado las teorías sobre la generación. En general, la idea de una oposición entre propuestas preformacionistas y epigenetistas resulta convincente desde un punto de vista estrictamente biológico ( $\mathrm{Ne}$ edham, 1934, p. 40). Cabe recordar que para el preformacionismo, la constitución del nuevo ser era el resultado de una información esencial e inmutable, preconfigurada por naturaleza en el óvulo femenino (ovismo), o en el esperma masculino (animaculismo), mientras que el epigenetismo mostró un carácter más dúctil y un sentido procesual, agregativo o incluso evolutivo, en el que la generación debía observarse como un proceso biológico marcado por la mezcla de sustancias seminales que se organizaban durante el proceso de gestación. Sin embargo, esta oposición no resulta igualmente convincente a la hora de afrontar el sentido antropológico que era inherente a la propia biología. Desde ese punto de vista tanto el carácter preexistente del alma dentro de la esencia humana, como el sentido agregativo que el comportamiento debía ejercer sobre la misma, fueron elementos clave para construir un razonamiento científico sobre las variaciones (Bossi, 2003).

Esta relación adquirió especial importancia dentro de la tradición cristiana. Durante el llamado Periodo Antiguo, las ideas sobre la generación propuestas por los Padres de la Iglesia admitieron cierto grado de predestinación en la configuración natural del ser humano, al tiempo que consideraron que el desarrollo correcto de sus facultades animales, incluida la propia generación, dependía del estado en el que se encontrara el alma de los agentes generadores, tanto en el momento de la concepción como en el proceso de gestación, lo que explicaba el carácter más marcado de las variaciones humanas (imagen2). Fue Agustín de Hipona quien sentó cátedra en este punto, al afirmar que mientras los engendrados por la virtud nacían "tocados por Dios" como "vasos de misericordia", los nacidos en pecado lo harían marcados por la iniquidad como "vasos de ira" (Hipona, 412-426, p. 998 y 1005) $)^{9}$. Su distinción, puramente preformacionista, se vinculó, no obstante, a una defensa del pecado original frente a distintas herejías que, como 
Imagen 2. La Trinidad introduce la preformación humana durante el coito. Imagen sacada de Mansel, Jehan (ca.1559). Livre lequel entre aultres matieres traitte de la nativité Nostre Seigneur Jhesu Crist. T2, p.1. Códice de la Bibliothèque del Arsenal. Paris.

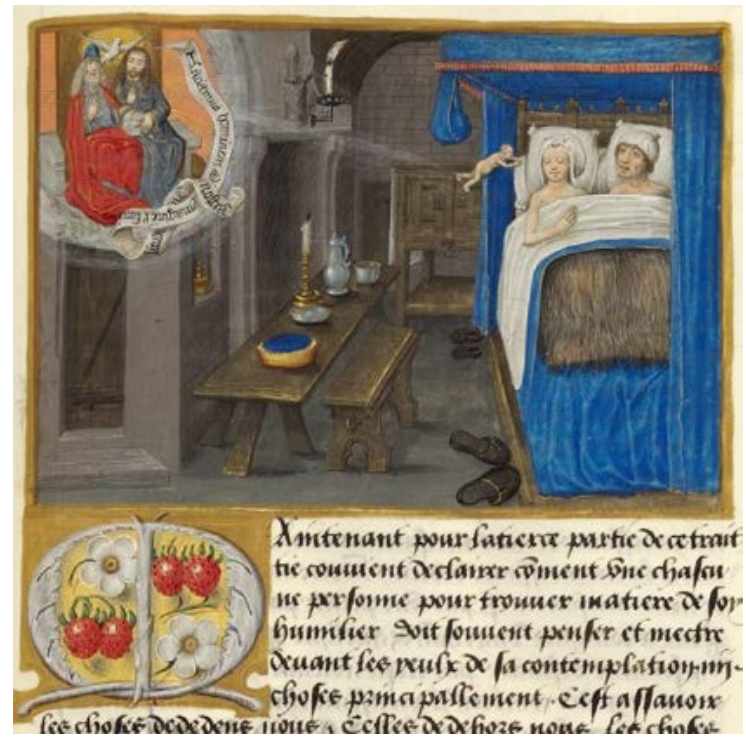

el pelagianismo, afirmaban que todos los seres nacían libres de las faltas y pecados de sus padres, siendo estos aprehendidos por la imitación de pautas culturales $^{10}$. Contrariamente a ello, el dogma agustiniano estableció que el pecado original era un elemento "natural", que desviaba la condición esencial del ser humano hacia un potencial netamente inferior al de sus antecesores, apoyándose en la fórmula "propagatione non imitatione", lo que significaba que todos morimos "por culpa del pecado de Adán" (Hipona, 412-426, Vol. II, Lib. XIII).

La inclusión del dogma del pecado original, hizo que la variación pasara a considerarse como el fruto de la transmisión diferencial de un potencial genésico, tan dependiente de la marca sensible que el pecado dejaba en los individuos, como de la reincidencia de éstos sobre él. Por su parte, la inclusión del libre albedrío, implicaba abrir la idea preformacionista de la predestinación a la interferencia de causas externas $y$, el reconocimiento de grados de probabilidad e incertidumbre dentro del proceso de génesis. Algunos autores como Avicena (ca. 1020, Trac. III, Lib. II), Averroes $^{11}$, Alberto Magno (ca. 1200, Lib. XXI) o Hildegard von Bingen (ca. 1150, Lib. II), identificaron momentos críticos en el proceso de gestación, cuya alteración produciría las variaciones en los individuos, llegando así a presupuestos claramente epigenéticos (ima- gen3). No obstante, siguieron imputando a los individuos un grado de humanidad directamente proporcional a su relación con Dios, potenciando así el principio del homo imago Dei ${ }^{12}$.

La racionalización de este principio permitió al cristianismo apropiarse de un tipo biológico "superior" propio del primer hombre ${ }^{13}$ y construir, a partir de él, un criterio fundamental de humanidad útil para reconocer la diferencia dentro de la variación. La vinculación de ese tipo original con el presupuesto de la acción de la divinidad, implicaba que toda relación variación-inferioridad podía explicarse por la conjunción entre el alma y el cuerpo. La idea que se instaló definitivamente con Tomás de Aquino fue que mientras el pecado original era transmitido como "pecado de naturaleza", y por tanto, marca de origen dentro de nuestra esencia, la transmisión del pecado de la persona requería de un hábito y, como tal, de un comportamiento moral. De este modo, el ejercicio del pecado pasó a entenderse como un elemento accidental para el cuerpo, y aunque el propio Tomás

Imagen 3. La representación el proceso de formación del alma durante la gestación del individuo. Pertenece a "El alma y su lugar" (Die Selle und ihr Zelt). Miniatura 5 (fol. 22) de Bingen, Hildegart von (ca. 1160). Liver Scivias. Codex Rupertsberger. Vid. (Saurma-Jeltsch, 1998, pp. 58-66)

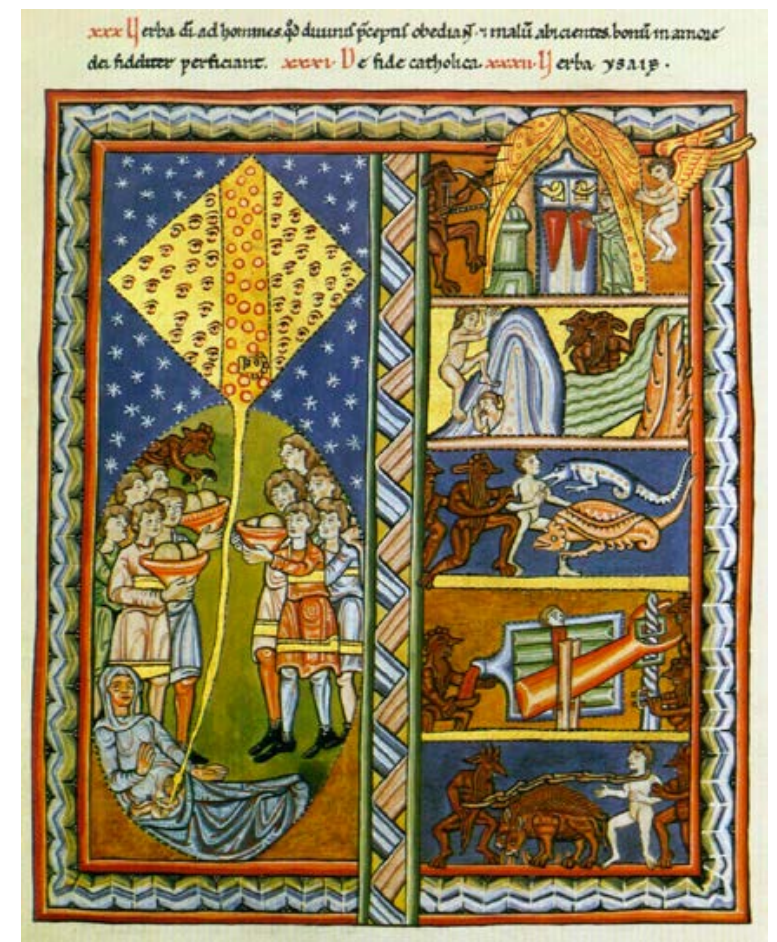


de Aquino reconociera que el acto en sí "no merece o desmerece a toda la naturaleza humana", la escolástica tomista no consideró su comisión como algo inocuo para la descendencia. Reproduciendo, punto por punto, la propuesta aristotélica del fenómeno de la generación, añadió varias cuestiones de relevancia, como que la perfección humana residía en la "virtud divina" otorgada por Dios en esencia y transmitida en la parte material por una "virtud formativa" que residía en las "razones seminales". También que la parte material o física del cuerpo debía ser completada por el alma que, aun siendo una entidad divina e imperfectible, era moldeable según el estado natural del cuerpo, por lo que se vería de algún modo condicionada por la incidencia del pecado original. De este modo, al igual que la virtud, el pecado residía en el alma, pero era

causado en el alma por el contagio del semen y está en él, es decir, en el semen, aunque no como un sujeto sino como la causa; por tanto, se transmite el pecado original por la transmisión del semen y no del alma (Aquino, ca. 1252, Dis. XVIII, Qua II).

La incidencia del pecado original se configuró así, como una inferioridad biológica cuya única solución radicaría en obtener "la gracia", un beneficio que Dios no otorgaba a cambio de nada:

de Cristo nacen los hijos de la gracia, no por la transmisión de la carne, sino por demérito de la acción: pero de Adán nacemos hijos de la ira por propagación, no por demérito (Aquino, ca. 1252, Dis. XX, Qua. II, art. III, resp. 3).

Sólo la fe en Cristo permitiría alcanzar el grado mínimo de perfección en esa relación, en la que la disposición del cuerpo condicionaba y era condicionada a un mismo tiempo por el alma. Por consiguiente, aquellos que no conocían a Cristo estaban destinados a cualquier forma de inferioridad, variable en la medida que la carencia de visión de Cristo, como la de visión de Dios, solo llegaría por dos medios, uno "privativo" por el que el propio Dios la niega (es decir, por castigo) y otro, más común, que implicaba un "defecto natural negativo" que era impropio de la naturaleza humana y estaba forzado por decisiones morales (Aquino, ca. 1252, Dis.XX, Qua. II, art. III).

\section{LA INMANENCIA DEL CONCEPTO DE PECADO $Y$ LAS DIFERENCIAS ENTRE TIPOS}

Como ya comentamos anteriormente, la convicción dentro del pensamiento cristiano de la existencia de una relación entre la moralidad y la constitución biológica de los individuos, condujo necesariamente a un relato monolítico sobre la condición humana. En líneas generales, igualdad y diferencia constituyeron caras de una misma moneda o, por ser aún más concretos, establecieron una rica dialéctica que fructificó en una variada red de pensamientos sobre la naturaleza humana (Stuurman, 2017). Dentro de los argumentos que apostaron por la diferencia, la paradoja del pensamiento racional cristiano en torno a las consecuencias biológicas del pecado original residió en que su capacidad original para extender la cualidad humana a todas las variaciones fue alimentada con una progresiva restricción de su calidad como "personas", estableciendo un criterio de exclusión que se endureció con el paso de los siglos.

Este fenómeno se observa en campos de conocimiento tan heterogéneos como la naturaleza de los monstruos (Boaistuau, 1564; Paré, 1575), la limpieza de sangre (Torrejoncillo, 1676) o el origen de los pueblos colonizados (Acosta, 1590; García, 1607), en los que abiertamente se planteó la diferencia de sus objetos como una variación biológica en grado de inferioridad con respecto al tipo original, estableciendo la causa probable de ese hecho en la ruptura con las normas de la moral o en una continuada reincidencia en el pecado ${ }^{14}$. No era preciso, por tanto, comprender los mecanismos fisiológicos de la transmisión hereditaria, para valorar que había un carácter consustancial entre la variación y el pecado, que se transmitía a la descendencia, en tanto que "generatio enim perversa est, et infideles filis" (Cruz, 1637, p. 56).

La explicación dogmática estableció líneas difíciles de franquear para el espíritu secularizador de la ciencia moderna, precisamente porque el dogma del pecado original ofrecía al pensamiento racional, no sólo una forma de catalogar las diferencias como rasgos de inferioridad ajustada a los intereses político-económicos de la época (Mignolo, 1999; 2011), sino también una forma de vincularlas con las acciones de los individuos, lo que informa sobre el sentido socioantropológico que la cultura estaba adquiriendo en occidente. Evidentemente, esta idea tuvo mayor recorrido dentro de una mentalidad epigenética cristiana, en la que la generación se planteaba como un proceso guiado por la conjunción de Dios y la naturaleza, sin embargo, ni la imposición del preformacionismo mecanicista a partir del siglo XVII, ni la consecuente desacralización y desmitificación del proceso de gestación, impidieron que la idea del pecado man- 
tuviera un valor principal a la hora de dirimir la relación entre variación, diferencia e inferioridad, como tampoco para entender la fuente y motivo por los que dicha inferioridad había sido infundida. Tal cambio de mentalidad se redujo a precisar cuándo era infundida y cuáles eran los factores que podían llegar a afectarla ${ }^{15}$.

Es por ello que preformacionistas como Gottfried Leibniz (1646-1716), advirtieron que, aunque la variación era un fenómeno preexistente a la reproducción de los seres vivos, nada podía producirse que no hubiera estado inscrito en la naturaleza desde su origen y "nada milagroso" actuaba en la génesis del ser humano "exceptuando el comienzo de estas cosas", pues "el organismo de los animales es un mecanismo que supone una preformación divina" (Leibniz, 1715-1716: 115). Desde esa misma perspectiva, Charles Bonet evaluó las diferencias entre especies, así como las de los individuos dentro de ellas, atendiendo a una "perfección relativa en el conjunto de los seres" a la que llamó "perfección mixta" ya que se definía por el grado de coordinación entre el alma y el cuerpo, una coordinación que era mayor en los humanos que en cualquier otro ser, a excepción de los ángeles y cuya presencia era en cualquier caso reminiscencia de $\operatorname{Dios}^{16}$.

Esta pervivencia de la fe resulta explícita en la teodicea racional de los siglos XVII y XVIII (imagen4), pero fue igualmente importante dentro de las líneas directrices que definieron al proyecto racional ilustrado, aunque de forma mucho más matizada ${ }^{17}$. Finalizando el siglo XVIII, la preexistencia en la reproducción de las formas fue puesta en duda por la experimentación con embriones, y una parte importante del pensamiento ilustrado recuperó la idea del proceso formativo, propia de las posturas epigenéticas, sin que ello implicara un cambio radical de paradigma, ya que, en muy amplio grado, la preexistencia de la forma siguió siendo necesaria para dotar de sentido al fenómeno.

Uno de los autores principales en este punto fue Pierre Louis Moreau de Maupertuis (1698-1759), quien consideró que el proceso de génesis estaba dominado por la existencia de elementos inmanentes que definían la "configuración interna" de los cuerpos, es decir, partes orgánicas "primitivas e incorruptibles" que se perpetuaban en todas las especies y a las que llamó "elemento similar". La idea, posteriormente desarrollada por Georges Louis Leclerc Buffon (1707-1788) en la "teoría del molde interior", reclamó
Imagen 4. La cadena de los seres. Grabado que ilustra el capítulo I del tratado Utriusque Cosmi maioris salicet et minoris metaphysica del médico inglés Robert Fludd (1617), T.1. Es interesante ver como la cadena, que une a Dios con la Naturaleza y a esta con el simio, no vincula directamente al hombre y la mujer, que aparecen encabezando el círculo de las criaturas naturales.

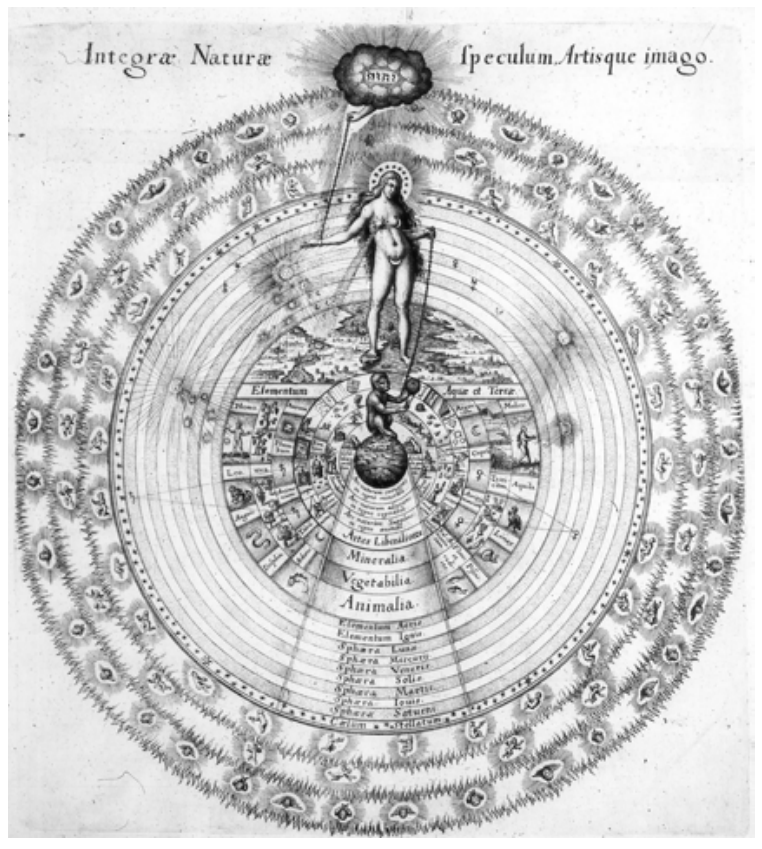

la existencia de una fuerza o "potencia activa" en los seres que de algún modo, contendría la información de las "partes originarias" del primer ser humano, y sería la encargada de reproducirlo "desde dentro", entendiendo por tal, no ya la simple ordenación de los órganos sino también, la correcta coordinación de las leyes físicas que regían sobre ellos, la forma en que éstos se desarrollaban y en la que finalmente se reproducían. Dichas partes originarias se consideraron una reminiscencia del primer tipo de la especie por lo que, en efecto, "el molde interior" era a un tiempo contenedor y replicante de la información del primer ser humano al que, por otro lado, se le reconoció un grado de perfección inmanente, esto es, una "unidad de tipo"18, que podía extrapolarse como grado de perfección de la especie.

Por más secularizado que se mostrara el razonamiento, su esencia seguía estando ligada a los límites de una "verdad" creacionista, sobre los que, reconocía abiertamente Maupertuis, "no se nos permite dudar", pues aquél que deseara postular "contra la 
historia del Génesis" encontraría cómo ésta "nos enseña que todos los pueblos de la tierra han salido de un único padre y una sola madre" (Maupertuis, 1745, p. 192). Ciertamente, la persistencia de Dios como elemento creador ya no implicaba una relación directa entre creador y criatura, por lo que, como ya avanzaba Leibniz, fuera cual fuera el objeto de la historia natural, el fenómeno de la variación debía analizarse a expensas de la actuación divina, marcado en todos los casos por la acción de las mismas fuerzas naturales. Esta premisa fue matizada por Buffon para el caso del ser humano, pues reconocía en él "una naturaleza muy diferente (...) tan superior a la de los brutos, que sería preciso ser tan irracional como ellos para confundirlos", por ello era preciso tener en cuenta "la historia más noble de su ser", esto es, la de su alma (Buffon, 1749, p. 14).

La importancia de la obra de Buffon radicó en su capacidad para sentar los fundamentos de la jerarquización de los individuos dentro de su especie. En su opinión, la variación era una alteración del tipo que conformó líneas de cambio superficial en relación a los tipos originales. Esta desviación del desarrollo "natural" del tipo, a la que llamó degeneración, se había producido por el acondicionamiento del cuerpo a causas externas como el clima o la alimentación, salvo en el caso del ser humano en el que fue una "extensión de nuestra naturaleza (que) no proviene tanto de las propiedades del cuerpo como de las del alma" (Buffon, 1766, pp. 249-250). De ahí que su materialización se marcara por una "pérdida de nuestro entendimiento" con respecto al del tipo original, así como de "la costumbre de emplearlo (que) ha permanecido sin ejercicio, en medio del tumulto de nuestras sensaciones corporales" (Buffon, 1749, p. 6).

Al vincular la modificación biológica del ser humano con aspectos culturales e intelectuales, Buffon comprendió la degeneración en base a patrones civilizatorios más que fisonómicos. Según su teoría, la primera civilización "digna de ese nombre, digna de nuestros respetos, como creadora de las ciencias y de las artes", se había situado en "las regiones septentrionales de Asia desde los 40 grados de latitud hasta los 55", zona que entonces se conocía como la Gran Tartaria (imagen5) (Buffon, 1778, pp. 303-304). Buffon afirmaba que, en un tiempo remoto, aquella zona había contado con unas condiciones climáticas que favorecieron el desarrollo de las condiciones físicas e intelectuales de sus habitantes, provocando un crecimiento inusitado de su cultura. Prueba de

Imagen 5. La Gran Tartaria, representada en el Atlas de Mercator y Hondius (1633), T.II, pp. 666-667. BNE.

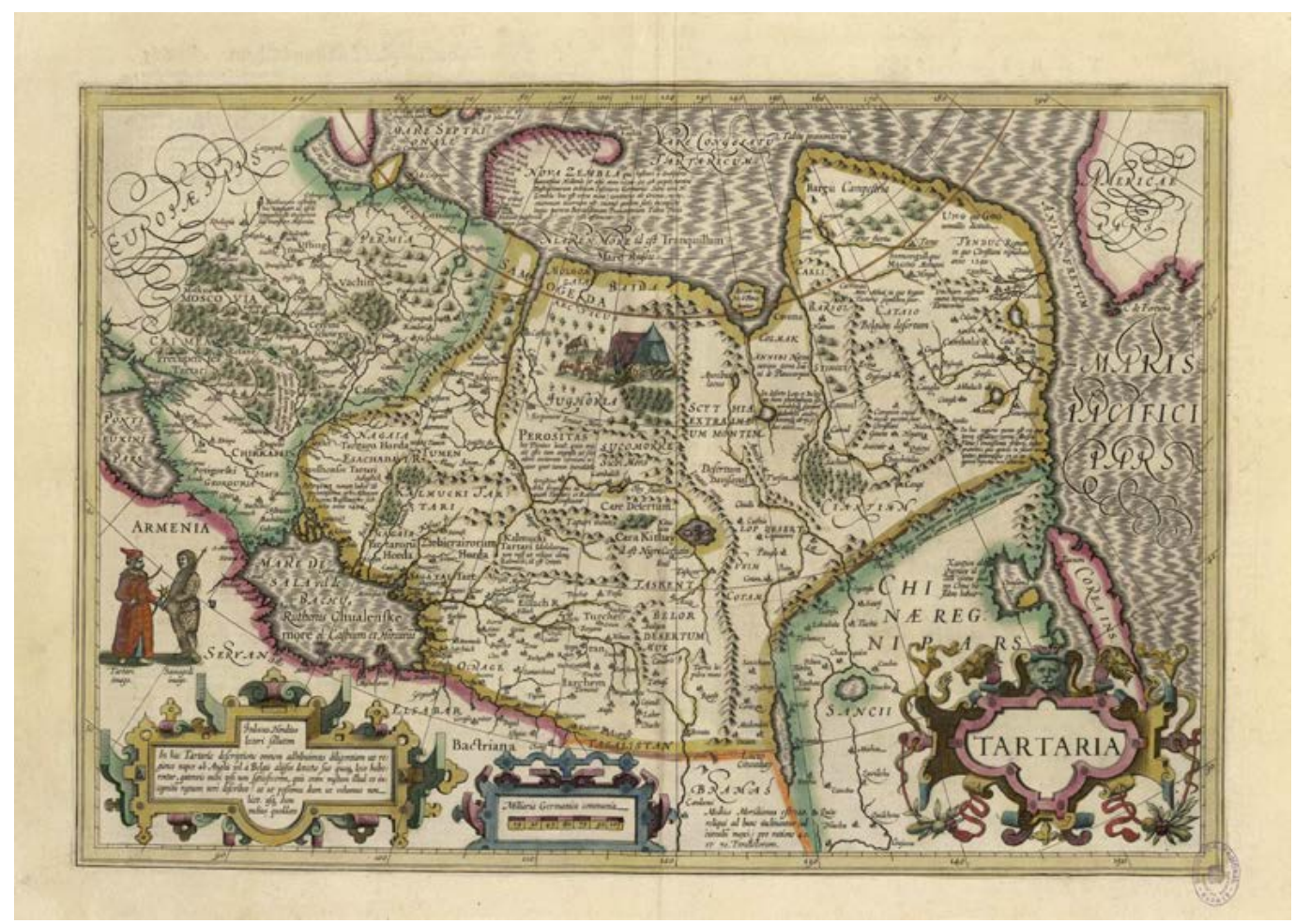


ello era que aquella civilización había teorizado el "periodo lunisolar de 600 años", que Giovanni Doménico Cassini (1625-1712) había "redescubierto" en (1689) y que, según el historiador Tito Flavio Josefo (ca. 38-101), ya habían conocido los patriarcas antediluvianos Mahalaleel, Jared, Enoc y Matusalén, hasta Noe (Josepho, ca. 93-94, s.p. Lib. I, Cap. III, F. 5-anv). En algún momento inmediatamente posterior a este último, las condiciones climáticas favorables desaparecieron y con ellas el equilibrio biológico y fisiológico especial que había provocado el desarrollo de su cultura, después de ello "las ramas estériles o degeneradas de este noble y antiguo tronco se extendieron por todas partes de la Tierra entre las naciones civilizadas" (Buffon, 1778, p. 313). Siguiendo al astrónomo Jean Sylvain Bailly (1775, pp. 109-128), Buffon dedujo que esa mediocridad científico-teórica comenzó a revertirse muchos años después cuando algunos grupos humanos iniciaron un lento y progresivo periodo de desarrollo de sus capacidades físicas y morales, que puso en línea ascendente a las ramas de la cultura de occidente, mientras que para el resto:

... a 30 siglos de luces quizá siguieron otros tantos de ignorancia. De todos estos bellos y primeros frutos del entendimiento humano no quedaron más que las heces (...) el hombre en fin sin educación, sin moral, reducido a pasar una vida solitaria y salvage (sic), no presenta en vez de su alta naturaleza, más que un ser degradado inferior al bruto (Buffon, 1778, pp. 313-314).

Si la naturaleza, y no Dios, era la única fuerza sobre la que cabía comprender el fenómeno de la variación del ser humano, la calidad de éste seguía midiéndose conforme a una constitución física y moral original que seguía requiriendo de la creación de Dios. Sólo así se podía concluir de modo racional que "el blanco es el color primitivo de los hombres", y que todas las demás variedades debían considerarse para la ciencia como "monstruos (...) desviaciones de la Naturaleza que no se preservan sino por el arte o por el régimen" (Maupertuis, 1745, pp. 189-190).

Al señalar la influencia del arte o el régimen sobre el tipo, la excepcionalidad de la variación en el ser humano quedaba ligada a aspectos de carácter moral, condicionando así las teorías en torno a la existencia de un elemento preformado en la naturaleza del ser, ya que, si como afirmaba Buffon, realmente había un "molde interior" que, de forma natural y bajo condiciones ambientales similares a las del origen, reproducía el estado de perfección esencial del primer tipo humano, era lógico pensar que, dado que la propia idiosincrasia de la naturaleza humana giraba en torno a la capacidad de los individuos para controlar la influencia del medio ambiente, la variación de su tipo era, al menos en cierta medida, un fenómeno autoinflingido, por lo que también lo era su degeneración. A lo largo del siglo siguiente, esta idea fue fundamental para evaluar las diferencias biológicas atribuibles al resto de las "variedades" no occidentales, pero sobre todo para catalogar la diversidad dentro de los propios tipos $^{19}$.

\section{LA FUERZA CREADORA Y LA SUPERIORIDAD BIOLÓGICA DEL HOMBRE EN OCCIDENTE}

Aunque el papel de Dios hubiera dejado de ser activo en la lógica de occidente sobre la variación, su poder seguía siendo en muchos sentidos determinante. La inmanencia de Dios como elemento creador, reforzó el peso que dentro de las causas secundarias debía otorgarse a las cuestiones morales, relativizando a su vez la influencia unívoca que se atribuía a los factores ambientales (Caponi, 2008). Esta resistencia a desprenderse de la idea de Dios como elemento creador bien pudo responder al difícil encaje teórico que las particularidades de la variación, y de su transmisión, tenían dentro de la visión holística y omnicomprensiva que tradicionalmente había mostrado la historia natural. Esta última disciplina consideraba la particularidad de las distintas especies y la forma en que los tipos variaban, como simples capítulos accidentales de un complejo libro en el que se narraba la filogenia del conjunto de los seres vivos (Roger, 1963, vid. Cap. IV).

Tal grado de desinterés por lo particular daría muestras de agotamiento desde finales del siglo XVIII, gracias a la confluencia de distintos factores. Por un lado, el desarrollo del estudio sobre los mecanismos que gobernaban las variaciones de los tipos, ligado al estudio fisiológico de la especie humana (Williams, 1994). Por otro lado, la aparición de teorías sobre la herencia de los caracteres biológicos (López Beltrán, 1992). Y, de modo general, por la progresiva individualización de los objetos de conocimiento natural, que dio lugar a "nuevas disciplinas" (Foucault, 1966). El interés por los mecanismos que regían los fenómenos naturales concretos, se tradujo en un análisis pormenorizado de la ontogénesis dentro de los tipos (Gould, 1977) y ligado a él por un restablecimiento teórico del vínculo entre las especies, animado por la recuperación del principio de la "escala natural" 
aristotélica, que impulsó los estudios sobre biología comparada (López Piñero, 1992, pp. 11-20).

Esta forma de desarrollo científico no impidió que la lógica dogmática del creacionismo monogenista siguiera aceptando que la superioridad del ser humano occidental emanaba de su mayor grado de cercanía con el creador, pero al menos abrió un espacio para la crítica. Algunos naturalistas como el médico escoces John Hunter (1728-1793) $)^{20}$, plantearon un modelo de gradación progresiva que, sin romper con el extremo de la idea original sobre la superioridad del tipo humano original, apostaba por dar una visión alternativa de su materialización (Hunter, 1786). Su idea, en general, afrontaba la supuesta relación entre la morfología y la perfección progresiva de las funciones orgánicas, invocando la recapitulación ontogenética como único patrón racional del que cabía colegir la "inferioridad" o "superioridad" de una especie sobre otra" ${ }^{21}$ es decir, que al "tomar una serie de animales de los más imperfectos a los perfectos, probablemente deberíamos encontrar un animal imperfecto que corresponda con alguna etapa del más perfecto" (Hunter, 1861, p. 204).

La apuesta de Hunter por explicar las diferencias desde un punto de vista secularizado era muy evidente. Su gradación en tipos inferiores y superiores, establecía que la materia embrionaria era un elemento reproductivo común para todas las especies y que, por tanto, el proceso natural de formación era idéntico para todos los seres vivos, al menos en sus fundamentos, ya que a partir de cierto punto el proceso de formación se complejizaba según el grado de perfección que mostrara la especie. Dicho desarrollo diferencial estaba marcado por el efecto de una fuerza o principio natural sensible, que conectaba con las partes del cuerpo, provocando la normalidad o la variación (Hunter, 1786, pp. 243-244). En este sentido, el grado de perfección de la criatura respondía a unos criterios puramente naturales, sin embargo, había dos aspectos que seguían sin explicarse, el primero y más evidente, el origen de esa fuerza natural que, de modo general, podría enlazarse con el principio de fuerza vital propio de las posturas animistas y vitalistas (Duchesneau, 1985), y que, a falta de una explicación mejor, siguió siendo vinculado con la acción de la divinidad. Así lo hizo, por ejemplo, el ya citado Charles Bonnet (1760), quien recurrió a la idea de una "fuerza creadora" como elemento diferenciador de los peldaños de su escala naturae. El segundo aspecto fue la perdurabilidad del criterio de perfec- ción en el tipo humano, con el añadido de que, al vincular su normalidad con el estado previo de la materia formativa, la variación y la diferencia dentro de los tipos pasaron a manifestarse como fenómenos de carácter patológico, cambio del que dio cuenta la obra de Johan Friedrich Blumenbach.

Asumiendo como ciertas las propuestas de Hunter, Blumenbach identificó el elemento "fuerza" con el concepto de nisus formativus, al que dotó de la función de crear el embrión convirtiendo la masa informe de la mezcla seminal en "materia madura". Asimismo, lo incluyó dentro del conjunto más amplio de "fuerzas vitales" y le atribuyó un papel determinante en la producción y reproducción de variaciones, en tanto que su efecto dependía de la condición previa de la materia seminal (Blumenbach, 1828) ${ }^{22}$. Al extrapolar este razonamiento natural hacia el estudio de la variación en la especie humana, Blumenbach terminó concluyendo ${ }^{23}$ que había cierto carácter patológico en toda variación ajena al patrón de perfección original, es decir, que "las razas y las variedades son desviaciones de las formas originales específicas de los cuerpos organizados de especies individuales, que resultan de su variación o degeneración gradual" producida por la exposición a causas externas. Un razonamiento que sin duda alguna se vio perfilado por la convicción de que "bajo cualquier principio fisiológico, la (raza) caucasiana debía considerarse como primaria o intermediaria" del resto de variedades (Blumenbach, 1788: ep. 15).

La diferenciación racial de Blumenbach posicionó las variaciones del ser humano dentro de la escala natural, fomentando una forma de pensamiento antropológico que, desde finales del siglo XVIII y hasta mediados del siglo XX, daría cabida a formas de determinismo biológico cada vez más radicales (imagen6). Sin embargo, la propuesta de Blumenbach no muestra esa forma de determinismo. El sentido general y constante con el que concebía el nisus formativus implicaba que todas las razas humanas eran variaciones del primer tipo formado, por lo que la degeneración debía entenderse como un fenómeno intrínseco al conjunto de la especie y no a una única raza. Por otro lado, la pertenencia al tipo racial superior no era un rasgo concluyente capaz de confirmar la superioridad del individuo. Sólo la materialización individual de la cultura que se asumía como propia de ese tipo, permitía establecer un juicio en ese aspecto, por lo que la existencia de individuos de las "razas inferiores" superiores a algunos individuos de 
Imagen 6. Cráneos de las cinco razas de Blumenbach, el caucásico aparece en el centro (3. feninae georgianae). Pertenece la figura II que ilustra las últimas páginas de (Blumenbach, 1781).

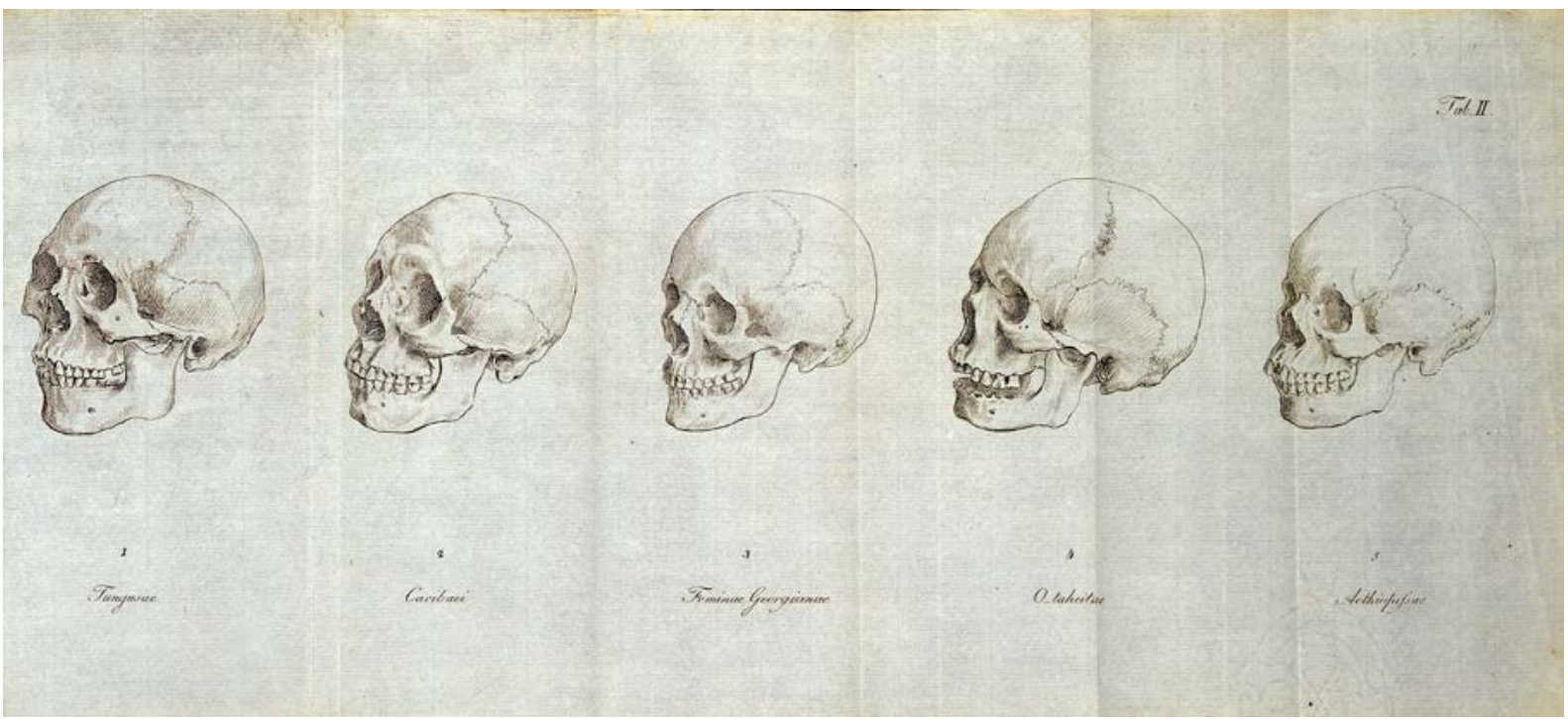

la raza superior, no sólo era posible, sino que además era bastante común (Blumenbach, 1828, p. 560). En cualquiera de los casos, dado el carácter constante de la fuerza formativa, el sentido patológico que Blumenbach imputó a las variaciones sólo podía radicar en la materia formativa, por lo que independientemente del grado de degeneración que la raza, o cualquier otra variación, produjeran sobre esa materia, siempre habría una marca de inferioridad con respecto al tipo original de la especie que sería reproducida en la descendencia. Fue precisamente ese punto el que derivó la cuestión hacia la transmisión de las variaciones.

\section{LA TRANSMISIÓN DE VARIACIONES Y EL SENTIDO MORAL DE LA HERENCIA}

Aunque finales del siglo XVIII no hubiera muchas dudas sobre la superioridad natural de los rasgos del tipo humano occidental, las dificultades para concretar el proceso fisiológico por el que una parte de ellos se reproducía en la descendencia, mientras que otra se perdía o variaba, era más que evidente. Incluso recurriendo a la idea de una fuerza formativa, que en esencia tampoco constituía un elemento cognoscible ni mesurable, el desarrollo teórico se había limitado a constatar la persistencia de unos "rasgos fuertes" (género, raza, familia) o herencia orgánica, cuya no reproducción se consideraba fuera de los límites naturales, mientras que la idea de variación se vinculaba con la transmisión de los rasgos accidentales o particulares (López Beltrán, 2004). Esta explicación, que funcionó excepcionalmente bien dentro de los límites del razonamiento natural fijista y creacionista $^{24}$, fue puesta en duda desde principios del siglo XIX por Jean Baptiste Lamarck (1809), al señalar que el estado actual de las especies debía concebirse como el resultado de una larga transmisión de caracteres adquiridos, dentro de cadenas de variaciones extendidas a lo largo de escalas de tiempo geológico, lo que no sólo ponía en duda la existencia de caracteres fijos o rasgos inmutables, sino que reducía a la mínima expresión cualquier valoración moral sobre las causas del cambio (Lamarck, 1801, p. 13) ${ }^{25}$.

La observación biológica del potencial de la herencia como transmisor de variaciones comenzó a tomar fuerza al analizar estas propuestas biológicas desde una perspectiva patológica. Desde el campo de la teratología, dedicada al análisis exhaustivo de los mecanismos fisiológicos que regían sobre las variaciones en el estado embrionario, el zoólogo francés Étienne Geoffroy Saint-Hilaire (1772-1844), planteó la existencia de un plan general de formación que se materializaba en un proceso fisiológico común de formación de los seres vivos, "un plan único, que siempre ha sido el mismo en esencia, pero que ha variado de mil maneras en sus partes accesorias" (Saint-Hilaire, 1796, p. 20). Aunque no ponía en duda la existencia de una fuerza generatriz que, originada en Dios, ani- 
mara dicho plan, consideró que éste estaba sujeto a normas naturales, reconocibles en el análisis de las irregularidades, lo que significaba que la variación no era fruto del azar ni de la preexistencia, sino el resultado de errores formativos que podían ser reproducidos y, por tanto, también previstos (Saint-Hilaire, 1818-1822, vol. II, p. 483).

Años después, su hijo Isidore Geoffroy Saint Hilaire, estableció una clasificación de esas variaciones en función de su gravedad, incluyendo entre ellas una serie de "anomalías simples poco graves", a las que llamó "vicios de conformación" (Saint-Hilaire, 18331836 , vol.I, p. 24). Dentro de ellas, consideró la existencia de "embranchemens anomaux dissimilaires" o variaciones disimilares que afectaban a las terminaciones de los vasos sanguíneos o del sistema nervioso (Saint-Hilaire, 1833-1836, vol.I, p. 341-342), cuya reproducción alcanzaba a un número indeterminado de generaciones cercano a las tres o cuatro ${ }^{26}$. Cuanto más simple, o menos visible, parecían ser esos "vicios", más evidente parecía también su transmisión por herencia biológica de ascendentes (Saint-Hilaire, 1833-1836, vol.I, p. 503). Dado que los individuos que sufrían estas variaciones apenas diferían del "estado normal" de su especie I.G. Saint Hilaire las separó de aquellas que afectaban a "las monstruosidades más grandes" (Saint-Hilaire, 1833-1836, vol.I, p. 22), derivando su reproducción al aún difuso campo de la herencia:

Es un hecho conocido en todo tiempo, como los padres, del mismo modo que transmiten a sus hijos su constitución física, sus características, e incluso sus cualidades morales e intelectuales, transmiten también sus defectos de organización dirigidos a una o varias partes de sus cuerpos (...) a veces sucede que (...) los males de un individuo terminan convirtiéndose en los de toda una raza. (...) La explicación completa de todos estos hechos está más allá del alcance de la ciencia actual...(Saint-Hilaire, 1833-1836, vol. III, p. 271).

Este tipo de variaciones genésicas resultó de especial interés para una parte de la medicina (López Beltrán, 1995). A finales del siglo XVIII, los médicos que, como Alexis Pujol (1787-1788) o Jean-François Pagès (1798), establecieron las primeras relaciones de enfermedades hereditarias, concluyeron la necesaria existencia de un nexo causal entre las propiedades biológicas de organismos ligados por relaciones genealógicas y la transmisión de caracteres determinados. Por conveniencia, sus propuestas se fundaron en principios análogos a los planteados por Blumenbach sobre la variación, aceptando una visión patológica de la herencia cuyo único sustento racional se limitaba a la disposición humoral supuesta a los ascendentes, una convicción que, a su vez, se veía reforzada por el reducido alcance de su objeto, limitado a las pocas generaciones de las que podían dar cuenta sus pacientes, así como por la correlación entre espíritu y materia planteada por un vitalismo fisiológico de marcada impronta cristiana (Barthez, 1778, Vol. I, pp. $120-121)^{27}$.

Al igual que ocurriera con la variación en biología, los médicos valoraron la herencia como un elemento fundamental en la transmisión de caracteres esenciales y significativo en la transmisión de caracteres adquiridos. No obstante, y por motivos obvios, los médicos prescindieron de los largos espacios de tiempo que marcaba la perspectiva biológica, amplificando el valor de la herencia dentro del proceso de variación. Esto, unido a falta de un conocimiento fisiológico acabado sobre los mecanismos de transmisión, llevó a muchos de ellos a sobrevalorar el carácter patológico de fenómeno, posición que llegó a extremarse cuando, iniciado el siglo XIX, la disciplina reivindicó su legitimidad para intervenir en la solución de diversos problemas sociales (López Beltrán, 1992; Vallejo, 2013).

En ese nuevo marco, parte importante del concepto patológico de la herencia se centró en el sentido fundamentalmente moral de sus causas. Autores como Petit, rechazaron que la herencia tuviera relación directa con causas ambientales externas o que estuviera necesariamente vinculada con un elemento esencial propio de una raza, para concebirla como "un germen" generado por las decisiones que los individuos tomaban con respecto a su forma de vida o su moral, que se materializaba en hábitos capaces de modificar su fisiología, provocando cambios (lesiones) en su cuerpo, potencialmente transmisibles y capaces de producir "la degeneración de linajes completos", poniendo en riesgo al conjunto de la sociedad. Si bien, al igual que cualquier otro germen, podía ser erradicada mediante una coerción higiénica (Petit, 1817, pp. V-VIII).

La contundencia ideológica de ese mensaje aumentó a partir de 1847, como consecuencia de la formulación de las "leyes de la herencia" del médico francés Prosper Lucas (1808-1885), según las cuales la naturaleza producía al mismo tiempo formas improvisadas, dando lugar a tipos originales (loi de l'invention), pero también imitaba sobre patrones previos (loi de l'imitation) subordinándose a una composición aná- 
loga a la de los tipos ya creados (Lucas, 1847-1850, vol. I, pp. 24-25). La preeminencia de uno u otro valor dependería de varios mecanismos que fueron construidos sobre los mismos principios fisiológicos de los que se sirvió I.G. Saint-Hilaire para postular la existencia de los "vicios de conformación" o ramificaciones "disimilares". La acción de alguno o varios de esos mecanismos era necesaria, por lo que siempre podría preverse, pero raramente podría concretarse, lo que seguía dejando abierto un amplio margen para la interpretación de sus causas. De este modo, en la configuración del nuevo ser influiría la herencia directa, si mostraba rasgos de los ascendentes inmediatos; herencia indirecta, si esos rasgos provenían de ascendentes colaterales; herencia de retorno si provenían de generaciones anteriores a la de los ascendientes inmediatos; o herencia por impregnación, en caso de que provinieran de cónyuges previos (Lucas, 18471850, vol. I, p. 2).

Durante un largo periodo de tiempo, la propuesta de Lucas satisfizo a una clase médica ávida por obtener unos criterios sólidos que permitieran explicar la variación patológica de los tipos, sin embargo, la imposibilidad de concretar una previsión de los mecanismos de la herencia a priori, hizo que dichos criterios se limitaran a canalizar "científicamente" el sentido moral e individualizador con el que tradicionalmente se había establecido la relación entre la variación, la diferencia y la inferioridad. El propio Lucas asumió y reforzó los fundamentos del creacionismo desde una posición católica, abiertamente crítica con el razonamiento transformista, dado que la diversidad de formas que generaba la herencia, no podía abrirse al "horizonte sin fin, sin luz, ni base" en el que la habían encerrado autores como Lamarck.

Coherentemente con su postura, Lucas siguió requiriendo de un tipo original perfecto sobre el que apoyar la existencia de las variaciones y la vinculación de ese tipo con un principio abstracto directo como la divinidad, o mediado como la fuerza natural, lejos de ser un problema para la razón, siguió pareciéndole la respuesta más lógica a las causas de la organización de los cuerpos, que sencillamente surgirían "como la fuerza y la ley de nuestro ser (...) si sus bases son abstractas, es porque son profundas como las de las preguntas que transportan la mente a grandes alturas". La diversidad seguía planteándose así, como una "intervención en acto o expresión orgánica en la vida", que penetraba en la uniformidad del primer tipo, produciendo una fuerza que lo alejaba de "lo similar" y que, transformando el modelo, lo reconfiguraba y lo devolvía sin salirse de los límites de la especie "inventando, en imitación incluso, por variedad, a la creación". Esto había provocado la escisión racial de la especie como variación esencial, pero no determinante, dado que "muchas de (las razas) son para nosotros tan viejas como la especie", siendo más determinantes "las variedades de seres que las componen". Variaciones individuales del tipo que, conforme al sentido de la fuerza que las había motivado, podrían llegar a convertirse en formas patológicas cuya mera existencia implicaba un riesgo.

\section{CONCLUSIONES}

Con las leyes de la herencia de Lucas, la inclusión de los principios dogmáticos que, conforme a la idea de un tipo creado, sostenían la relación entre la variación y la diferencia, permitiendo justificar la desigualdad dentro del campo de la ciencia, alcanzaron el grado de desarrollo necesario para prescindir del recurso a la acción de una fuerza sobrenatural dentro del proceso. Aún en el punto más oscuro de su obra, marcado por el carácter disimilar de los mecanismos hereditarios y la consiguiente imposibilidad de establecer un análisis científico a priori de los mismos, la lógica de dichos mecanismos invitaba a pensar que necesariamente debían estar regidos por principios naturales, sobre los que poco tenía que ver el designio divino, pero para cuya prevención sería preciso realizar una evaluación moral del individuo, cuestión que pasaba a formar parte de la responsabilidad del médico. Ello explica el inestimable valor que tendría la obra de Lucas para el desarrollo de la psiquiatría durante la segunda mitad del siglo XIX (Huertas García-Alejo, 1987) pero también, de modo más general, el marcado determinismo biológico con el que comenzó a interpretarse la problemática social en los años siguientes (Pick, 1989; Huertas García-Alejo, 1998).

Dentro de este giro biológico de lo social, la inmanencia del dogma de la creación en la construcción científica de la relación entre variación, diferencia y desigualdad, se observa claramente en la teoría de la degeneración, que le dio inicio. Su promotor, el médico Benedict Auguste Morel, se basó en las leyes de Lucas para dotar de una historia natural a aquellas variaciones que consideró como tipos humanos patológicos, individuos "paralelamente distintos", seres 
"degenerados", cuyo origen radicaba en "las nuevas condiciones que debió crear en el hombre el gran acontecimiento del pecado original" (Morel, 1857, p. 2) y cuya persistencia hasta el presente debía explicarse por su alejamiento de la "ley de Dios", pues

La ley moral (...) es una, es universal, es verdadera, y da la posibilidad a todos de aceptarla y practicarla, ofreciendo una prueba certera de la unidad de la especie, de la que se puede deducir la unión y difusión entre las distintas razas humanas. (...) estas razas, no han sido todas alcanzadas por un mismo grado de civilización, y en el seno de las propias naciones civilizadas, existen las clases caídas, que apenas entrevén el movimiento ascendente de las clases superiores, a las que no pueden acceder si son abandonadas a su suerte. (...) la aplicación del tratamiento moral a estas masas desheredadas se presenta como uno de los más nobles, pero también de los más difíciles sujetos de estudio a los que pueden aspirar los verdaderos amigos de la humanidad (Morel, 1857, p. 4).

Morel fue uno de los últimos hombres de ciencia que, de modo explícito, recurrió al dogma del homo imago dei para seguir justificando la inferioridad por la relación entre la variación y la diferencia. Dos años después, la aparición de la obra de Darwin pondría en duda los principios del creacionismo clásico imponiendo la idea de la adaptación como fundamento lógico de la evolución y variación humana (Darwin, 1859; 1868), mientras que la recuperación de la obra de Mendel (1866) a principios del siglo XX, acabaría con el ya bastante cuestionado dogma de la trasmisión generacional del pecado. Pero incluso tras la implantación de estas nuevas perspectivas, la pervivencia del dogma de la creación, dentro de las propuestas sobre la superioridad biológica occidental aún mantendría una amplia influencia en la ciencia desde un punto de vista ideológico y moral, cuestión esta que impone un tipo de análisis que no se ajusta a los objetivos precisos de nuestro artículo.

Llegado este punto, es preciso concluir que una amplia mirada hacia la inmanencia del principio dogmático del homo imago dei dentro del pensamiento racional, permite valorar su influencia no como una forma de pensamiento científico, sino como una forma de acceso al saber que emanó de una percepción cultural extendida sobre nuestro origen, y que ejerció una influencia trascendental en la construcción de la ciencia, cuya presencia va mucho más allá del grado de aceptación que cada autor, en cada época, mostrara hacia el principio. Parece igualmente evidente que su uso generalizado no respondió tanto a la ne- cesidad de explicar la diferencia, como a su utilidad para reclamar un sentido autorreferencial de la superioridad frente al diferente. De este modo, ningún arma ha sido tan afilada, tan destructiva, ni tan útil a los intereses de su dueño como la que, defendiendo la igual naturaleza humana, atribuye a las diferencias evidentes de sus términos un grado de desigualdad latente que trasciende lo accidental para convertirse en esencial y permanente, justificando así su inferioridad y el derecho a su sometimiento.

La construcción de esta forma de razonamiento siempre precisó de un elemento irracional más o menos evidente, la idea de una causa eficiente o Dios creador cuya perfección quedaría reflejada en la obra fue difuminada, naturalizada y reformulada por un pensamiento científico secular, pero raramente fue cuestionada, sino que más bien fue adecuada al sentir de cada época, dándole acomodo dentro de diversas ideas y teorías como el molde interior, la unidad de tipo, la fuerza formativa o la escala natural, cuyo sentido teleológico siguió aferrándose a formas de razonamiento similares, permitiendo extender ese principio de superioridad sobre fundamentos más sólidos.

La asociación de ese razonamiento con el ejercicio del poder fue igualmente un elemento de continuidad, que sirvió a la ciencia y la política para reafirmarse mutuamente. El análisis en un espacio de tiempo dilatado nos permite, sin embargo, matizar la relación entre ambos términos. La progresiva naturalización de la idea de la superioridad occidental, no fue consecuencia de las formas de poder establecido, sino que más bien ejerció como motor de ellas, reafirmando progresivamente el papel del científico hacia posiciones de poder, que no nacen de un ejercicio objetivo de la ciencia sino de su capacidad de mediación. Resulta sintomático a este respecto que los argumentos dirigidos a señalar la superioridad biológica de occidente raramente fueron acompañados de una defensa explicita de los ejercicios de poder que sustentaban al colonialismo y que, no obstante, las ideas y teorías esgrimidas permitieran justificar en cada caso el sentido general de aquellos procesos, del mismo modo que permitieron legitimar el ejercicio del poder sobre los individuos por parte de los nuevos estados liberales. A lo largo de la segunda parte del siglo XIX, las ideas que, fundadas en un sentido determinista de la ciencia, reconocieron como necesaria la imposición de unos individuos sobre otros, ya fuera señalando superioridad de una raza sobre el resto, la 
necesidad de someter a todo un sexo o la lógica natural de un orden social injusto, siguieron poniendo en evidencia la persistencia de una idea que nunca fue ciencia y que no obstante siempre dominó en la ciencia: que existía una superioridad biológica origi- nal, que había sido otorgada por un ente superior y que los pocos elegidos que contaban con ella, tenían el derecho y el deber de extenderla, por todos los medios.

\section{NOTAS}

1 La crítica sobre la construcción de la diferencia por parte de la ciencia ha tenido un desarrollo muy marcado especialmente tras la publicación de Foucault (1961) y posteriores trabajos en los que se analiza la patologización moderna de la diferencia vid. (Foucault, 2000). Autores como Stephen Jay Gould (1981) analizaron la construcción científica y vulgarización de ese concepto y más recientemente ha pasado a analizarse desde distintas categorías, principalmente género y raza. Vid. p.e. (Ellison y Goodman, 2006; Fuechtner; et al., 2018).

2 Un análisis de su desarrollo y utilidad puede consultarse el capítulo introductorio de la reciente obra de Christiansen y Jensen, (2019). Por otro lado, su vigencia tiene una clara representación en trabajos como los de Smith (2015) o Davies (2016).

3 Esta visión, más progresiva o incluso positiva de la naturaleza humana, ha sido quizá la más analizada por la historiografía (Tuveson, 1949; Maravall, 1966 o Gray, 2007).

4 Al respecto de la multiplicidad de argumentos y de los recursos para la construcción de los mismos, conviene revisar la obra de Joan-Pau Rubiés, especialmente (Rubiés, 2000).

5 La más conocida es la de Los trabajos y los días de Hesíodo (ca. 700 BCE: 106-200), la idea tiene reminiscencia clara en las dos edades de Platón (ca. 362 AEC: 269a-273e) y sobre todo en las cuatro edades de Ovidio (ca. 8, Lib.I: 75-150)

6 La reflexión sobre el mito de la creación ofrece, en ambas tradiciones, una amplia variedad propuestas no siempre coincidentes con las que terminarían siendo dominantes. En la tradición clásica las obras de Anaxágoras de Clazómenas o Tales de Mileto buscaron las causas de la génesis original en fenómenos estrictamente naturales llevando al extremo la máxima de que "nada puede ser generado de la nada" (Fuente Freyre, 2002, pp. 42-45). La falta de sentido de esa búsqueda es puesta en evidencia en Fedón (Platón, ca. 387 BCE, p. 82), donde se induce la necesidad de buscar un principio inmaterial como generador de la esencia de los seres. Por su parte, dentro de la cultura hebrea no es raro encontrar propuestas que apostaran por una creación mediada y no directa, que contrastan con el relato dominante del judaísmo y el cristianismo (Graves y Patai, 1963, pp. 65-71).

7 Las principales teorías genésicas de la tradición grecolatina estuvieron marcadas por el modelo hipocrático de pangénesis, que hablaba de un líquido seminal constituido por información reunida de todo el cuerpo, transmitida por las

venas y los nervios hasta los genitales, donde era eyaculado durante el acto sexual (Hipócrates, ca. 420-400 BCE: 1 , p. 247) y por la propuesta aristotélica del $\pi \varepsilon \rho ı \tau \tau \dot{\mu} \mu \alpha \tau \alpha$ o semen peritomático, construido como un residuo latente de la alimentación, que fluye por el organismo como parte de la sangre, y en el que se contiene la información necesaria para "duplicar" al individuo (Aristóteles, ca. 347-335 BCE, lib. I, 724b-725a). La influencia de ambas es evidente, por ejemplo, en Galeno (ca. 164-175, lib. XIV).

8 La idea de la transmisión en el sentido "genético", con el que hoy la entendemos como "herencia biológica", no se produciría hasta finales del siglo XIX (López Beltrán, 2004, pp. 41-44).

9 La idea de Dios como alfarero y el hombre como "vaso" o recipiente, responde a la metáfora usada por Pablo de Tarso en (Rom. 9:21-23), para señalar las dos cualidades de hombre los "preparados para la destrucción" que son fruto de su ira y los "preparados de antemano para la gloria", que serían el fruto de su misericordia. Por otro lado, etimológicamente hablando el concepto ira,-ae, viene del indoeuropeo eis- que viene a significar "mover rápidamente o pasión", lo que encajaría aún más con el significado pretendido en la obra de Agustín, quien ejemplifica a ese tipo de hombres con Ismael, el hijo engendrado en adulterio por Abraham y su esclava Agar, que al contrario que Isaac (engendrado por la gracia de Dios con su legítima mujer Sara), estaba sujeto al doble pecado, carnal y original. Hemos consultado la traducción de Santos Santamaría y Miguel Fuentes en (Hipona, 412-426), (Lib. XV; cap. 2), vol. XVII, p. 144, 998 y 1005.

10 Según el propio Agustín (ca. 418) el problema de la propuesta pelagianista no radicaba en la negación del pecado original, sino en negar éste como causa de la muerte de Adán y por tanto del resto de la especie humana. En general la idea, planteada por el monje britano Pelagio (ca. 410), advertía que la transmisión generacional del pecado original se daba por la imitación del pecado de los padres y no por una forma de transmisión de carácter biológico.

11 La idea de la generación como proceso la muestra Averroes de modo general en sus comentarios sobre la generación de los animales de Aristóteles (ca. 347-335 BCE), vid. (Averroes, ca. 1189). Previamente a ello se observan anotaciones en este sentido dentro de las Kulliyyât (Averroes, ca. 1162: 27-28).

12 Alberto Magno tomó de Averroes la idea de que en cuestiones de generación "la naturaleza no hace nada sin la dirección de las inteligencias superiores". Vid. (Scoti, 1655, p. 32). 
13 La superioridad biológica de ese tipo tiene un fundamento moral en tanto que se consideraba "en cierto sentido, poseedor de todas las virtudes (...) pues había (en él) una rectitud tal, que la razón estaba sometida a Dios; y las potencias inferiores a la razón" (Aquino, 1265-1274, vol. 1, C. 95/a.3)

14 La variación de posturas con respecto a la cuestión de la colonización fue analizada en profundidad por Giuliano Gliozzi (1977). Un ejemplo característico de una visión distinta sobre el origen genésico de los seres humanos y su influencia determinante sobre las diferencias y la inferioridad de algunos pueblos es la famosa obra de La Peyrère sobre los preadamitas (La Peyrère, 1655). Por su parte, la importancia de estas diferencias ha sido valorada por Sánchez Villa (2017). Asimismo, los distintos argumentos con respecto al poder del bautismo en la regeneración del alma y sus consecuencias para el cuerpo, fue analizada en profundidad dentro del seminario dirigido por Adriano Prosperi sobre la historia del bautismo (Prosperi, 2006).

15 Las diferencias entre epigenetismo y preformacionismo serían muy marcadas, sobre todo en lo que se refiere a las implicaciones ideológicas que subyacen a ambos modelos, así como en el consiguiente concepto político. No obstante, la continuidad entre ambos modelos, marcada por los principios del creacionismo es advertida por autoras como Jennifer Mensch (2013) o Clara Pinto-Correia (1997).

16 La idea, claramente tomada de Tomás de Aquino, se desarrolla en Bonnet (1760, Cap. VII).

17 Horacio Capel Sáez (1982) incidió en esa relación entre ciencia y religión en las ciencias naturales de los siglos XVII y XVIII. Sobre la influencia que ejerció el razonamiento dogmático en el pensamiento ilustrado (Haakonssen, 1996; Arana Cañedo-Argüelles, 1999; 2007; Sorkin, 2008)

18 Técnicamente, la expresión "unidad de tipo" no aparece en la obra de Buffon, pero se popularizó esta interpretación gracias a su alumno Jean Pierre Flourens (1844, pp. 89, 164 y sig.).

19 Sobre la primera de estas evaluaciones, dio cuenta el propio Buffon en un largo repaso sobre la inferioridad de los distintos pueblos del planeta en base a su alejamiento del patrón de perfección localizado en la mezcla del hombre blanco y la tradición cultural europea (Buffon, 1749). La propuesta de Buffon dio lugar a la aparición de diversas obras en el mismo sentido. Las más famosas fueron las de Cornelius de Pauw (1770) y Guillaume Raynal (1770), dirigidas a señalar la inferioridad del pueblo latinoamericano. La polémica que generaron estas obras es analizada en la obra de Antonello Gerbi (1955), quien señala la oposición con respecto a las posiciones de autores como Alexander von Humbold, Francisco Javier Clavijero o Johann Wolfgang von Goethe.

20 Sobre John Hunter interesan los capítulos de François Duchesneau e lan Rolfe dedicados a anatomía y fisiología en la obra editada por Bynum y Porter (1985, pp. 259-322), que trata sobre sobre su hermano, William Hunter.

21 Ciertamente, la idea de una gradación progresiva de especies sobre criterios de inferioridad y superioridad remite a la obra de William Harvey (Harveo, 1651), Ex. 27, pp. 86-87, pero fue desarrollada en la obra de (Hunter, 1786), p. 295. En el prefacio de la reedición de aquel trabajo en 1837, el médico Richard Owen atribuyó a Hunter estas palabras: "si tomáramos una serie de animales, del más imperfecto al perfecto, encontraríamos probablemente un animal imperfecto correspondiendo con algún estado del más perfecto" (p.XXVI), es por ello que Moore (2005), p. 498, incluyó a Hunter dentro del grupo de "descubridores" de la Teoría de la Recapitulación. Gould (1977), pp. 27-28, rechaza dicha inclusión.

22 El concepto nisus formativus o Bildungstriebes (fuerza formativa o impulso formativo) abrió la obra principal de Blumenbach (1781a, pp. 1-2), que recogía el texto de su tesis doctoral que leída en 1775 . El concepto fue desarrollado posteriormente (Blumenbach, 1780; 1781b).

23 Como es sabido, en las distintas ediciones de De generis humani varietate nativa que aparecieron entre $1775 \mathrm{y}$ 1795 , Blumenbach introdujo cambios importantes con respecto al número de razas, que pasaron de cuatro a cinco, y también en lo concerniente al sentido patológico de las variaciones, que no se plantea hasta (Blumenbach, 1781a, pp. 51-53). Sobre esta cuestión vid. (López Beltrán, 2004).

24 La dominancia de dicho razonamiento se debió en gran medida al peso del razonamiento natural de Cuvier (1817). Su influencia tuvo amplio desarrollo el pensamiento paleontológico y antropológico del siglo XIX (Pelayo López, 1999; Blanckaert, 2000).

25 Vid. (Bourdier, 1972).

26 Esta cifra se valoraba en base a los datos de distintos autores, entre ellos Maupertuis (1745) quien no precisaba generaciones de modo exacto, sino una valoración probabilística que suponía el caso de deformidad de un hombre con seis dedos, en $1 \times 20.000$ y la probabilidad de que se diera esa "monstruosidad" en tres generaciones consecutivas en $1 \times 8$ billones, (vid. pp. 277-278). Asimismo, citaba un breve artículo de un cirujano (Renou, 1774), que afirmaba que la transmisión de estos defectos era más común de madres a hijos y de padres a hijas. (Saint-Hilaire, 18331836, vol.I, p. 496.)

27 Sobre este sentido en el vitalismo vid. (Williams, 1994). 


\section{BIBLIOGRAFÍA}

Acosta, José De (1590), Historia natural y moral de las Indias. Madrid: Casa de Alonso Martín. 1608.

Alter, Robert (1996), Genesis. Translation and Commentary. London. W.W. Norton and Co.

Aquino, Tomás De (ca. 1252), Comentario a las sentencias de Pedro Lombardo Vol. II/1. La Creación: Ángeles, seres corpóreos, hombre: EUNSA. 2005.

Aquino, Tomás De (1265-1274), Suma de Teología. V vols. Madrid: BAC. 2001.

Arana Cañedo-Argüelles, Juan (1999), Las raíces ilustradas del conflicto entre fe y razón. Madrid: Encuentro.

Arana Cañedo-Argüelles, Juan (2007), “Ciencia y Religión en la Ilustración Francesa". En: José Montesinos y Sergio Toledo (eds.), Ciencia y Religión en la Edad Moderna, pp. 273-290. La Orotava: Fundación Canaria Orotava de Historia de la Ciencia.

Aristóteles (ca. 347-335 BCE), Reproducción de los animales. Madrid: Gredos.

Averroes, (Abû L'Walîd Ibn Rushd) (ca. 1162), El libro de las generalidades de la medicina. Madrid: Trotta. 2003.

Averroes (ca. 1189), Epítome del libro sobre la generación y la corrupción. Madrid: CSIC. 1992.

Avicena, (Ibn Sîna) (ca. 1020), Avicennae Arabum Medicorum Principis. Canon Medicinæ. Venetiis (Venecia): Apud luntas. 1595.

Barthez, Paul-Joseph (1778), Nouveaux éléments de la science de l'homme. 2 vols. Montpellier: Chez Jean Martel.

Bingen, Hildegard Von (ca. 1150), Causae et Curae. Lipsiae: Aedibus B.G. Teubneri. 1903.

Blanckaert, Claude (2000), “Avant Adam. Les représentations analogiques de l'homme fossile dans la première moitié du XIXe siècle". En: Albert Ducros y Jaqueline Ducros (eds.), L'Homme préhistorique. Images et imaginaire. Paris: L'Harmattan, pp. 23-61.

Blumenbach, Johann Friedrich (1780), “Über den Bildungstrieb (Nisus formativus) und seinen Einfluß auf die Generation und Reproduction". Göttingisches Magazin der Wissenschaften und Litteratur. 1 (5), pp 247-266.

Blumenbach, Johann Friedrich (1781a), De generis humani varietate nativa. Goettingae: apud Vidvam.

Blumenbach, Johann Friedrich (1781b), Über den Bildungstrieb und das Zeugungsgeschäfte. Göttingen: Johann Christian Dieterich.

Blumenbach, Johann Friedrich (1788), Handbuch der Naturgeschichte. Göttingen: Dieterich'schen Buchhandlung. 1825

Blumenbach, Johann Friedrich (1828), Elements of Physiology. London: Longman.

Boaistuau, Pierre (1564), Historias prodigiosas y maravillosas de diversos sucesos acaecidos en el Mundo. Medina del Campo: Francisco del Canto. 1586.
Bonnet, Charles (1760), Essai analytique sur les facultés de l'âme. Copenhague: Chez les Freres.

Bossi, Laura (2003), Historia natural del alma. Madrid: Machado. 2015.

Bourdier, Franck (1972), "Lamarck et Geoffroy Saint-Hilaire face au problème de révolution biologique". Revue d'histoire des sciences. 25 (4).

Buffon, (Leclerc, Georges-Louis) (1749), “De la naturaleza del hombre". En: Renato Ricardo Castel (ed.), Compendio de la Historia Natural de Buffon. (vol.III) 21vol. Madrid: Imprenta de Villalpando. 1802-1808, pp. 5-144

Buffon, (Leclerc, Georges-Louis) (1766), “De la degeneración de los animales". En: René-Richard de Castel (ed.), Compendio de la Historia Natural de Buffon, (vol.IV) 21vol. Madrid: Imprenta de Villalpando. 1802-1808, p. 245 y 335.

Buffon, (Leclerc, Georges-Louis) (1778), “Las Épocas de la Naturaleza". En: René-Richard de Castel (ed.), Compendio de la Historia Natural de Buffon. (vol.II) 21 vol. Madrid: Imprenta de Villalpando. 1802-1808.

Bynum, W.F.; Porter, Roy, eds. (1985), William Hunter and the eighteenth-century medical world. Cambridge University Press. 2002.

Capel Sáez, Horacio (1982), La física sagrada. Creencias religiosas y teorías científicas en los orígenes de la geomorfología española. Barcelona: Serbal.

Caponi, Gustavo (2008), “Unidad de tipo y degeneración en la Historia Natural de Buffon". Filosofía e Historia de la Biología. 3, pp. 179-194

Cassini, Giovanni Domenico (1689), “Régles de l'Astronomie Indienne pour calculer les mouvements du soleil et de la lune". En: Recueil d'observations faites en plusieurs voyages par ordre de sa Majesté, pour perfectionner l'astronomie et la geographie. Paris: Imprimerie Royale. 1693, pp. 1-64

Cesaire, Aime (1955), "Discurso sobre el colonialismo (fragmento)". Latinoamérica. Cuadernos de cultura Latinoamericana, n. 54 (Sep. 1979), pp 1-27.

Christiansen, Christian Olaf; Jensen Steven L. B. (2019), “Histories of Global Inequality: Introduction." En: Christiansen, Christian Olaf; Jensen Steven L. B (eds.), Histories of Global Inequality. New Perspectives. Cham: Palgrave MacMillan, pp. 1-32.

Cruz, Gerónimo de la (1637), Defensa de los estatutos y noblezas españolas: destierro de los abusos, y rigores de los informantes. Zaragoza: Hospital Real y General de nuestra Señora de Gracia.

Cuvier, Georges Léopold C. F. D. (1817), Regne animal distribué d'après son organisation. 3 vols. Paris: Chez Deterville.

Darwin, Charles R. (1859), On the Origin of Species. London: John Murray.

Darwin, Charles R. (1868), The Variation of Animals and Plants under Domestication. 2 vols. London: John Murray. 
Davies, Surekha (2016), Renaissance, Ethnography and the Invention of the Human. Cambridge: Cambridge University Press.

Duchesneau, François (1985), "Vitalism in late eighteenthcentury physiology: the cases of Barthez, Blumenbach and John Hunter". En: W.F. Bynum y Roy Porter (eds.), William Hunter and the eighteenth-century medical world. Cambridge University Press, 2002, pp. 259-296.

Durand, Gilbert (1978), "Pérennité, dérivations et usure du mythe". En: Danielle Chauvin (ed.), Champs de l'imaginaire. Grenoble: ELLUG. 1996, pp. 81-108.

Ellison, George; Goodman, Alan H., eds. (2006), The Nature of Difference. Science, Society and Human Biology s.l.: Taylor \& Francis.

Estrada Díaz, Juan A. (2000), "Del primado del cosmos al descubrimiento de la subjetividad interior". En: Pedro Gómez García (coord.), Las ilusiones de la identidad. Madrid: Cátedra, pp. 181-204.

Flourens, Jean Pierre (1844), Buffon. Histoire de ses travaux et de ses idées. Paris: Paulin.

Foucault, Michel (1961), Historia de la locura en la época clásica. 2 vols. Buenos Aires. México D.F.: FCE. 1982.

Foucault, Michel (1966), Las palabras y las cosas. Una arqueología de las ciencias humanas. México DF: Siglo XXI. 1972.

Foucault, Michel (2000), Los Anormales: curso en el Collège de France (1974-1975). Buenos Aires: FCE.

Fuechtner, Veronika; Haynes, Douglas E.; Jones, Ryan M., eds. (2018), A Global History of Sexual Science, 1880-1960. Oakland: University of California Press.

Fuente Freyre, José Antonio De La (2002), La biología en la Antigüedad y la Edad Media. Universidad de Salamanca.

Gadamer, Hans-Georg (1960), Verdad y método. 2 vols. Salamanca: Sígueme. 1998.

Gagné, Renaud (2013), Ancestral Fault in Ancient Greece. Cambridge University Press.

Galeno (ca. 164-175) (2010), Del uso de las partes. Madrid: Gredos.

García, Gregorio (1607), Origen de los Indios de el Nuevo Mundo, e Indias Occidentales. Valencia: Casa de Pedro Patricio Mey.

Gerbi, Antonello (1955), La disputa del Nuevo Mundo. Historia de una polémica 17050-1900. México: FCE.

Gliozzi, Giuliano (1977) Adamo E II Nuovo Mondo. La Nascita Dell'antropologia Come Ideologia Coloniale: Delle Genealogie Bibliche Alle Teoria Razziali (1500-1700). Firenze: La Nouva Italia Editrice.

Gould, Stephen Jay (1977), Ontogenia y filogenia. Ley fundamental biogenética. Barcelona: Crítica. 2010.

Gould, Stephen Jay (1981), La falsa medida del hombre. Barcelona: Crítica. 2010.

Gould, Stephen Jay (1999), Ciencia versus religión. Un falso conflicto. Madrid: Crítica. 2000.
Graves, Robert; Patai, Raphael (1963), Los mitos hebreos. Buenos Aires: Losada. 1969.

Haakonssen, Knud (1996), "Enlightened Dissent: an introduction". En: Knud Haakonssen (ed.), Enlightenment and Religion. Rational Dissent in Eighteenth-Century Britain. Cambridge University Press, 2006, pp. 1-11.

Harveo, Guilielmo (Harvey, William) (1651), Exercitationes de Generatione Animalium. Londini: Typis Du-Gardianis.

Herrero de Jáuregui, Miguel (2007), Tradición órfica y cristianismo antiguo. Madrid: Trotta.

Hesíodo (ca. 700 BCE), Los trabajos y los días. Madrid: Aguilar. 1964.

Hipócrates (ca. 420-400 BCE), "Sobre la Generación”. En, Tratados Hipocráticos VIII. Madrid: Gredos, 2003, pp. 247-257.

Hipona, Agustín de (412-426), "La ciudad de Dios (Edición Bilingüe)". En: Obras Completas de San Agustín. Madrid: BAC. 2000.

Hipona, Agustín de (ca. 418), "Contra las dos epístolas de los pelagianos". En: Obras de San Agustín en edición bilingüe. Tratados sobre la gracia II. Madrid: BAC. 1952, pp. 457-671.

Huertas García-Alejo, Rafael (1987), Locura y degeneración: psiquiatría y sociedad en el positivismo francés. Madrid: CSIC.

Huertas García-Alejo, Rafael (1998), Clasificar y educar. Historia natural y social de la deficiencia mental. Madrid: CSIC.

Hunter, John (1786), Observations on certain parts of the animal œconomy. London: s.e. 1837.

Hunter, John (1861), Essays and observations on natural history, anatomy, physiology, psychology and geology. 2 vols. s.l.: John van Voorst.

Josepho, Flavio (ca. 93-94), Los Veynte Libros de las Antiguedades ludaycas. Angers: Martín Nucio. 1554.

Kennedy, Rebeca F.; Jones-Lewis, Molly, eds. (2016), The Routledge Handbook of Identity and the Environment in the Classical and Medieval Worlds. Abingdon: Routledge.

Kuhn, Thomas (1962), La estructura de las revoluciones cientificas. Mexico D.F.: FCE. 1971

La Peyrère, Isaac (1655), Præadamitæ. Sive Exercitatio Super Versibus Duodecimo, Decimotertio, Decimoquarto, Capitis Quinti Epistolæ D. Pauli Ad Romanos. Quivus Inducuntur Primi Homines Ante Adamum Conditi. s.l.: s.e.

Lamarck, Jean Baptiste P.A. (1801), Système des animaux sans vertèbres. Paris: Muséum d’Hist. Naturelle.

Lamarck, Jean Baptiste P.A. (1809), Philosophie Zoologique. 2 vols. Vol. I. Paris: Chez L'Auteur.

Leibniz, Gottfried Wilhelm (1715-1716), “Quinta carta de Leibniz". En: Eloy Rada (ed.), La polémica Leibniz-Clarke, pp. 99-136. Madrid: Taurus. 1980.

López Beltrán, Carlos (1992), Human Heredity (1750-1870). The Construction of a Scientific Domain. King's College London. Tesis doctoral. 
López Beltrán, Carlos (1995), "«Les maladies héréditaires». 18 th century disputes in France". Revue d'histoire des sciences. 48 (3), pp. 307-350.

López Beltrán, Carlos (2004), El sesgo hereditario. Ámbitos históricos del concepto de herencia biológica. México D. F.: UNAM.

López Piñero, José María (1992), Anatomía comparada antes y después del darwinismo. Madrid: Akal.

Lucas, Prosper (1847-1850), Traité philosophique et physiologique de l'hérédité naturelle. 2 vols. Paris: J.B. Baillière.

Magno, Alberto (ca. 1200), De Animalibus Lib. XXVI. Lyon: Cludi Prost. Petri \& Claudii Rigard. 1651.

Maupertuis, Pierre Louis (1745), Venus Physique. s.I.: s.e.

Mendel, Gregor Johann (1866), "Verscuche über PflanzenHybriden". Verhandlungen des Naturforschenden Vereines in Brünn. IV (1865), pp. 20-52.

Mensch, Jennifer (2013), Kant's Organicism. Epigenesis and the Development of Critical Philosophy. Chicago. London: University of Chicago Press.

Mercator, Gerard; Hondius, Jodocus (1633), Atlas, ou représentation du monde universel et des parties d'icelui. 2 vols. Amsterdam: H. Hondius.

Mignolo, Walter (1999), The Darker Side of the Renaissance. Literacy, Territoriality, and Colonization. Ann Arbor: University of Michigan Press. 2001.

Mignolo, Walter (2011), The Darker Side of Western Modernity: Global Futures, Decolonial Options. Durham: Duke University Press.

Moore, Wendy (2005), The Knife Man. London: Bantam.

Morel, Bénédict A. (1857), Traité des dégénérescences physiques, intellectuelles et morales de l'espèce humaine et des causes qui produisent ces variétés maladives. Paris: J.B. Baillière.

Needham, Joseph (1934), A History of Embryology. Cambridge University Press. 2014.

Ovidio (ca. 8), Metamorfosis. 2 vols. Vol. I (Libros I-V). Madrid: Gredos. 2008.

Pagès, Jean François (1798), "Hereditaires". En: Encyclopédie Méthodique. Paris: Chez H. Agasse, pp 160-176

Paré, Ambroise (1575), Monstruos y prodigios. Madrid: Siruela. 1987.

Pauw, Cornelius de (1770), Recherches philosophiques sur les Américains. 2 vols. Berlin: s.e.

Pelagio (ca. 410), "Incipiy ad Romanos". En: Alexander Souter (ed.), Pelagius's Expositions of Thirteen Epistles of St. Paul. Cambridge University Press. 1929, pp. 9-126

Pelayo, Francisco (1999), Ciencia y creencia en España durante el siglo XIX. La paleontología en el debate sobre el darwinismo. Madrid: CSIC.

Peset, José Luis (1983), Ciencia y marginación. Sobre negros, locos y criminales. Barcelona: Crítica.
Petit, Marie Antoine (1817), Essais sur les maladies héréditaires. Paris: Chez Gabon, Libraire.

Pick, Daniel (1989), Faces of Degeneration. A European disorder, c.1848-c.1918. Cambridge University Press.

Pinto-Correia, Clara (1997), The Ovary of Eve. Egg and Sperm and Preformation. Chicago: The University of Chicago Press.

Platón (ca. 362 AEC), "Político". En: Diálogos (V). Madrid: Gredos. 1988 , pp. 483-617.

Platón (ca. 387 BCE), "Fedón". En: Patricio de Azcárate (ed.), Obras completas de Platón. Madrid: Medina y Navarro. 1871, pp. 19-112

Plinius Secundus, Gaius (77-79 ap.), Historia Natural. Libros VII-XI. Madrid: Gredos. 1995.

Prosperi, Adriano, ed. (2006), Salvezza Delle Anime, Disciplina Dei Corpi. Un Seminario Sulla Storia Del Battesimo. Pisa: Edizioni della Normale.

Pujol, Alexis (1787-1788), "Essai sur les Maladies Héréditaires". En: François Gabriel Bouisseau (ed.), CEuvres diverses de médecine practique d'Alexis Pujol, pp. 211-420. Castres: F. Gausy. An 10. (1801).

Raynal, Guillaume-Thomas-Françoise (1770), Histoire philosophique et politique des établissements et du commerce des Européens dans les deux Indes. 6 vols. Amsterdam: s.e.

Renou, Joseph-Etienne (1774), "Observation sur quelques families sex-digitaries du Bas-Anjou". Observations sur la Physique, sur l'Histoire Naturelle et sur les Arts. IV (Novembre), pp. 372-374.

Roger, Jacques (1963), Les Sciences de la vie dans la pensée française au XVIIle siècle. Paris: Armand Michel. 1993.

Rousseau, Jean-Jacques (1754), Discurso sobre el orígen y los fundamentos de la desigualdad de condiciones entre los hombres. Madrid: Imprenta de José del Collado. 1820.

Rubiés, Joan-Pau (2000), Travel and Ethnology in the Renaissance South India through European Eyes, 1250-1625. Cambridge: Cambridge University Press, 2004.

Saint-Hilaire, Étienne Geoffroy (1796), “Mammifères. Memoire sur les rapports naturels des Makis Lemur, L. et Description d'une espèce nouvelle de Mammifere". Magasin Encyclopédique, ou Journal des sciences, des lettres et des arts. I (7), pp. 20-50.

Saint-Hilaire, Étienne Geoffroy (1818-1822), Philosophie Anatomique. 2 vols. Paris: J.B. Baillière.

Saint-Hilaire, Isidore Geoffroy (1833-1836), Histoire générale et particulière des anomalies de l'organisation chez l'homme et les animaux. 3 vols. Bruxelles: Hauman, Cattoir et Ca. 1837.

Sánchez Villa, Mario César (2017), "Monsters of an Awakened Reason. The Influence of the Biblical Account in the Development of the Scientific Theories on the Origin of Man and Its Variations." Culture \& History. 6: 1. https://dx.doi. org/10.3989/chdj.2016.020.

Saurma-Jeltsch, Liselotte E. (1998). Die Miniaturen im "Liber Scivias" der Hildegard von Bingen. Die Wucht der Vision und 
die Ordnung der Bilder. Wiesbaden: Dr. Ludwig Reichert Verlag.

Scoti, Michaelis (1655), Alberti Magni de Secretis Mulierum Libellus. Amsterdam: s.e.

Sorkin, David Jan (2008), The Religious Enlightenment. Protestants, Jews, and Catholics from London to Vienna. Princeton University Press.

Smith, Justin E. H. (2015), Nature, Human Nature, \& Human Difference. Princeton: Princeton University Press.

Stuurman, Siep (2017), The Invention of Humanity. Equality and Cultural Difference in World History. Cambridge, Harvard University Press.
Sylvain Bailly, Jean (1775), Histoire de l'Astronomie ancienne, depuis son origine jusqu'à l'établissement de l'Ecole d'Alexandrie. Paris: Chez de Bure. 1781.

Torrejoncillo, Francisco de (1676), Centinela contra Judíos, puesta en la torre de la Iglesia de Dios. Pamplona: s.e. 1720.

Vallejo, Mauro Sebastián (2013), “El problema de la herencia en la medicina francesa (1800-1846)". Llull. 36 (77), pp. 133-157.

Williams, Elizabeth A. (1994), The physical and the moral. Anthropology, physiology, and philosophical medicine in France, 1750-1850. Cambridge University Press. 